\title{
Knockdown of the differentially expressed gene TNFRSF12A inhibits hepatocellular carcinoma cell proliferation and migration in vitro
}

\author{
TAO WANG, SICONG MA, XINGXING QI, XIAOYIN TANG, DAN CUI, \\ ZHI WANG, JIACHANG CHI, PING LI and BO ZHAI \\ Department of Interventional Oncology, Renji Hospital, School of Medicine, \\ Shanghai Jiaotong University, Shanghai 200127, P.R. China
}

Received September 8, 2015; Accepted September 20, 2016

DOI: $10.3892 / \mathrm{mmr} .2017 .6154$

\begin{abstract}
Human hepatocellular carcinoma (HCC) has been reported to be highly insensitive to conventional chemotherapy. In the current study, the Agilent Whole Human Genome Oligo Microarray $(4 \mathrm{x} 44 \mathrm{~K})$ was used in order to identify the differentially expressed genes between HCC and adjacent tissues, and the top 22 differentially expressed genes were confirmed through reverse transcription-quantitative polymerase chain reaction. Among the identified differences in gene expression, expression of tumor necrosis factor receptor superfamily member 12A (TNFRSF12A) was markedly higher in HCC tissue than in adjacent tissue. Previous studies have suggested that TNFRSF12A may serve a role in tumor growth and metastasis, thus in the current study, TNFRSF12A was knocked down in the SMMC7721 cell line through siRNA. This demonstrated that cells exhibited reduced reproductive and metastatic capacity ex vivo. Thus, the results of the current study suggest that TNFRSF12A may be a candidate therapeutic target for cancer including HCC, and additional genes that exhibited significantly different expression from normal adjacent tissues require further study.
\end{abstract}

\section{Introduction}

Hepatocellular carcinoma (HCC) is a malignant tumor with one of the highest rates of mortality worldwide, and its associated morbidity and mortality remain of significant concern. In accordance with the World Health Organization GLOBOCAN database, HCC was the sixth most common cancer in 2012

Correspondence to: Dr Bo Zhai, Department of Interventional Oncology, Renji Hospital, School of Medicine, Shanghai Jiaotong University, 1630 East Road, The New Pudong, Shanghai 200127, P.R. China

E-mail: zhaiboshi@sina.com

Key words: TNFRSF12A, hepatocellular carcinoma, SMMC7721 cells
(782,000 new cancer cases worldwide, $5.6 \%$ of the total) and the second major cause of cancer death in $2012(746,000$ deaths, $9.1 \%$ of the total) (1).

Measuring levels of tumor biomarkers for HCC is an important tool for disease management and treatment, as elucidating factors that regulate cancer resistance and metastasis may aid in the development of effective strategies to treat and potentially cure cancer. A previous study established that the combination of three tumor markers, $\alpha$-fetoprotein (AFP), AFP-L3, and des- $\gamma$ carboxyprothrombin results in good predictive ability for patient survival following diagnosis (2). In addition, a recent study reported that Forkhead box M1 overexpression promotes HCC cell proliferation by cell cycle regulation (3). Numerous factors participate in liver carcinogenesis, including deregulation of microRNAs (miRNAs) (4); for example, miRNA-142-3p and miRNA-143-5p have been identified to be downregulated in HCC and exhibit synergistic effects on cell motility (5), with the epigenetic profile also observed to be changed in human HCC (6). Global gene expression profiles aid in the understanding of the transcriptomic landscape and molecular mechanism of HCC. In order to systematically study the transcriptomic differences between HCC and adjacent tissues, and elucidate the molecular mechanisms underlying HCC, the Agilent Whole Human Genome Oligo Microarray (4x44 K) was used, and reverse transcription-quantitative polymerase chain reaction (RT-qPCR) was used to screen and confirm the differentially expressed genes between $\mathrm{HCC}$ and normal adjacent tissues.

Tumor necrosis factor (TNF) receptor superfamily member 12A (TNFRSF12A; also known as CD266, FN14 and TWEAKR), is the smallest member of the TNF superfamily of receptors that lacks the cytoplasmic death domain (7), and has been reported to be elevated in various types of cancer, including HCC. TNFRSF12A overexpression in $\mathrm{HCC}$ has additionally been correlated with poor surgical outcome (8). TNFRSF12A has been previously identified to be upregulated in alcoholic hepatitis and is increased in experimental models of liver injury (9). It is additionally considered as a factor that promotes prostate cancer bone metastasis (10) and ectopic expression of TNFRSF12A increases the invasive activity of prostate cancer cells (11). In various cancer cell lines, including 
gastric cancer cell lines, TNFRSF12A has been observed to be upregulated during 5-fluorouracil (5-FU) treatment, which promotes resistance to 5-FU (12). Although TNFRSF12A has been reported to serve an important role in the development of various types of cancer, progression and resistance, howver the underlying molecular mechanisms in HCC remain to be fully elucidated. In the current study, TNFRSF12A was knocked down in the SMMC7721 cell line through siRNA. Cells in which TNFRSF12A was knocked down exhibited reduced reproductive and metastatic capacity ex vivo. These results were consistent with previous reports, for example Zhou et al $(13,14)$ reported that immunotoxins targeting the TNFRSF12A receptor can induce melanoma cell necrosis, suggesting that TNFRSF12A may be a candidate therapeutic target for cancer including HCC.

Thus, the present study aids in the systematic investigation of the molecular mechanism underlying $\mathrm{HCC}$, and additional genes which exhibit significantly different expression levels between normal adjacent tissues and HCC tissues.

\section{Materials and methods}

Tissue specimens and cell lines. The current study was approved by the ethics committee of Renji Hospital, Shanghai Jiao Tong University School of Medicine (Shanghai, China). A total of 20 patients (12 male, 8 female) with confirmed case of liver disease form Renji Hospital between July 2014 and July 2015 were included in the current study and the average age was $58.8 \pm 1.2$ years, the cancerous liver tissues were obtained during surgery. Written informed consent was obtained from each patient prior to the use of their tissue sample for scientific research. Tumor and adjacent liver tissues from surgical specimens were frozen in liquid nitrogen immediately until use. The human HCC cell line, SMMC7721, was obtained from the American Type Culture Collection (Manassas, VA, USA) and was cultured in complete RPMI-1640 medium containing 10\% fetal bovine serum (FBS; No-Worries ${ }^{\mathrm{TM}}$; Thermo Fisher Scientific, Inc., Waltham, MA, USA) and $100 \mathrm{U} / \mathrm{ml}$ penicillin/streptomycin (Invitrogen; Thermo Fisher Scientific, Inc.) at $37^{\circ} \mathrm{C}$ in a humidified atmosphere of $5 \% \mathrm{CO}_{2}$.

Microarray expression analysis. Total RNA from each sample was quantified using the NanoDrop ND-1000 (Thermo Fisher Scientific, Inc.) and the RNA integrity was assessed using standard denaturing agarose gel electrophoresis. For microarray analysis, the Agilent array platform was used. The sample preparation and microarray hybridization were performed based on the manufacturer's standard protocols. Briefly, total RNA from each sample was amplified and transcribed into fluorescent cRNA with using Agilent Quick Amp Labeling protocol (version 5.7; Agilent Technologies). The labeled cRNAs were hybridized onto the Whole Human Genome Oligo Microarray (4x44K; Agilent Technologies, Inc., Santa Clara, CA, USA). Subsequent to washing of the slides with potassium permanganate, the arrays were scanned using the Agilent Scanner G2505C (Agilent Technologies, Inc.).

Agilent Feature Extraction software (version 11.0.1.1; Agilent Technologies, Inc.) was used to analyze the acquired array images. Quantile normalization and subsequent data processing were performed using GeneSpring GX software, version 11.5.1 (Agilent Technologies, Inc.).

Reverse transcription-quantitative polymerase chain reaction (RT-qPCR). Total RNA was isolated using TRIzol (Life Technologies; Thermo Fisher Scientific, Inc.) according to the manufacturer's instructions. Extracted RNA was quantitated using NanoDrop ND-2000 (Thermo Fisher Scientific, Inc.) and treated with DNase I (Life Technologies; Thermo Fisher Scientific). The reverse transcription reaction was performed using PrimeScript ${ }^{\mathrm{TM}}$ RT Master Mix (Takara Biotechnology Co., Ltd., Dalian, China). RT-qPCR was performed on a Applied Biosystems 7500 machine using SYBR Green PCR Master Mix (Life Technologies; Thermo Fisher Scientific, Inc.). GAPDH was used as a loading control. The $\Delta \Delta \mathrm{Cq}$ method was used to calculate the fold change in the expression of each gene (15).

siRNA transfection in SMMC7721 cells. A non-targeting siRNA control [negative control (NC); cat. no. D-001320-01-50] was purchased from ABgene (Thermo Fisher Scientific, Inc.). TNFRSF12A-homo-400 (si-TNFRSF12A; cat. no. Q000007132-1-A) was purchased from Guangzhou RiboBio Co., Ltd. (Guangzhou, China). Cells were transfected using Lipofectamine 2000 in OptiMEM (Life Technologies; Thermo Fisher Scientific, Inc.). The knockdown of TNFRSF12A was quantified $48 \mathrm{~h}$ post-transfection using western blotting.

Western blot analysis. Western blot analysis was used to assess TNFRSF12A protein reduction. Cells were harvested in RIPA lysis buffer containing $50 \mathrm{mM}$ Tris, $\mathrm{pH}$ 7.4, $150 \mathrm{mM}$ $\mathrm{NaCl}, 1 \mathrm{mM}$ EDTA, 1\% Triton N-100, 1\% sodium deoxycholate and $0.1 \%$ sodium dodecyl sulfate and protease inhibitor cocktail (Roche Diagnostics, Basel, Switzerland) 48 h after siRNA transfection. The protein concentration was quantified using the Bio-Rad DC protein assay kit (Bio-Rad Laboratories, Inc. Hercules, CA, USA). Equal amounts of protein were separated using $12 \%$ SDS-PAGE and transferred to nitrocellulose membranes. The membranes were blocked with $5 \%$ nonfat dried milk in Tris-buffered saline, $\mathrm{pH} 8.0$, with $0.1 \%$ Tween 20 for $1 \mathrm{~h}$. The primary antibodies used included rabbit anti-human TNFRSF12A (Abcam, Cambridge, UK) and $\beta$-tubulin (R\&D Systems, Inc., Minneapolis, MN, USA). Membranes were washed with SuperSignal West dura (Thermo Fisher Scientific, Inc.) prior to visualizing the protein bands under the imaging apparatus (GE Healthcare Life Sciences, Chalfont, UK). Densitometric analysis was performed with ImageJ version 2.1.4.7 (National Institutes of Health, Bethesda, MD, USA).

Cell growth assay. Cells were plated into 96-well plates at a density of $3 \times 10^{3}$ cells/well and were maintained at $37^{\circ} \mathrm{C}$ in a humidified incubator. Subsequent to the initial transfection with the different siRNAs, cell growth was measured at 24,48 and $72 \mathrm{~h}$ by adding $20 \mu \mathrm{l} /$ well MTT $(5 \mathrm{mg} / \mathrm{ml}$; Sigma-Aldrich; Merck Millipore, Darmstadt, Germany). Plates were then incubated for an additional $4 \mathrm{~h}$ at $37^{\circ} \mathrm{C}$. Culture medium was removed from the wells, then dimethyl sulfoxide 
A

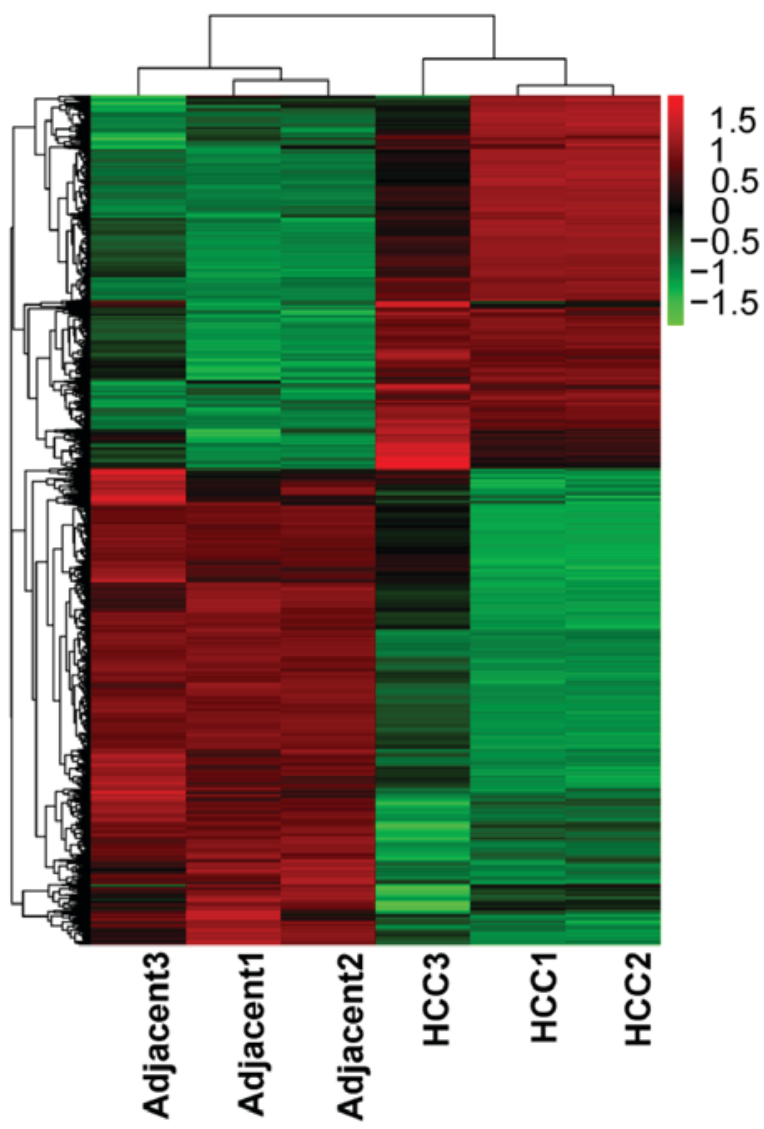

B

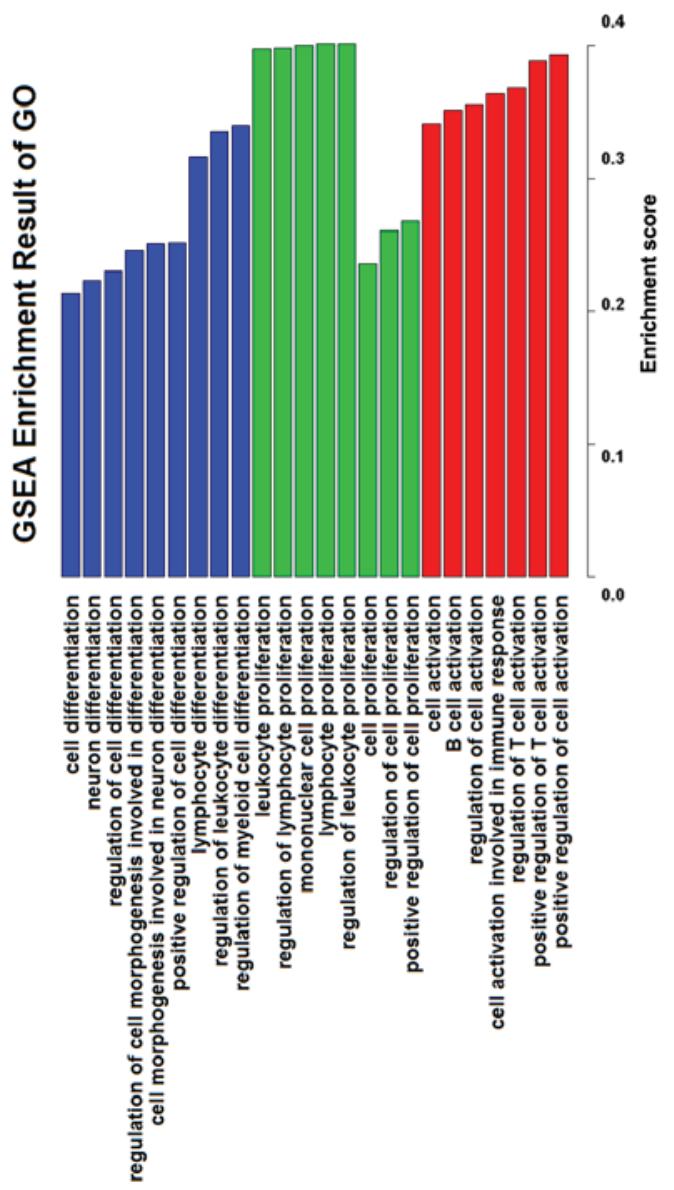

Figure 1. The heatmap and GO analysis between HCC and adjacent tissue. (A) Cluster results for all genes. (B) GO enrichment results by GSEA. GO, gene ontology; HCC, hepatocellular carcinoma; GSEA, gene set enrichment analysis.

(Sigma-Aldrich; Merck Millipore) was added at $100 \mu \mathrm{l} /$ well. Plates were then incubated for $20 \mathrm{~min}$ in a $37^{\circ} \mathrm{C}$ incubator, and absorbance was measured with the microplate reader (Bio-Rad Laboratories, Inc.) at a wavelength of $495 \mathrm{~nm}$. Each experiment was repeated three separate times.

Transwell migration assay. Micrometer pore size translucent transwell migration chambers (BD Biosciences, Franklin Lakes, NJ, USA) pre-coated with $50 \mu 1$ Matrigel (50 $\mu \mathrm{g}$ Matrigel in Dulbecco's modified Eagle's medium; Corning Incorporated, Corning, NY, USA) were plated in a 24-well plate. The lower chamber was filled with medium containing $10 \%$ FBS as a chemoattractant, and after transfection with the different siRNAs for $24 \mathrm{~h}, 1 \times 10^{5}$ cells were transferred to the upper chamber in $100 \mu \mathrm{l}$ serum-free medium. Following incubation for 24,48 and $72 \mathrm{~h}$ at $37^{\circ} \mathrm{C}$ in an environment with $5 \% \mathrm{CO}_{2}$, each transwell migration chamber was removed and 0.1\% crystal violet (Sigma-Aldrich; Merck Millipore) was used to stain the cells that passed through the filter. Cells adhering to the bottom of the chamber were counted under a microscope (Olympus Corporation, Tokyo, Japan) in a minimum of three random fields. The mean number of cells was obtained in each group.

Statistical analysis. Statistical analyses of the differences between groups were performed using Student's t-test. SPSS version 18 (SPSS, Inc., Chicago, IL, USA) was used to perform statistical analyses. $\mathrm{P}<0.05$ was considered to indicate a statistically significant difference.

\section{Results}

Heatmap and gene ontology (GO) analysis between HCC and adjacent tissues. Subsequent to gene expression normalization, the genes identified to be significantly different between $\mathrm{HCC}$ and adjacent tissues by the t-test were selected, and a heatmap was used to calculate a cluster result, (see Fig. 1A). Through the heatmap, the difference of the two sample types was evident. The two types of samples were separated into 2 clusters, and the differentially expressed genes also generated 2 groups. These results indicate that the gene expression data was reliable, thus the gene expression data was used for the subsequent steps of analysis.

Which functions and features are involved in and serve an important role in HCC is important, thus Gene Set Enrichment Analysis (GSEA) was conducted, focussing on functions in GO biological processes. Enrichment results from GSEA are presented in Fig. 1B. Several main features were selected in ordered by enrichment score. The main enrichment results were the functions of activation, proliferation and differentiation, and these functions predominantly serve a positive role in regulation. Thus, it is suggested that in the process 
Table I. Gene expression profiling of HCC and adjacent samples.

\begin{tabular}{|c|c|c|c|c|c|c|}
\hline Gene & $\mathrm{HCCl}$ & HCC2 & HCC3 & Adjacent1 & Adjacent2 & Adjacent 3 \\
\hline HSD11B1 & 11.76468 & 11.3246 & 11.54463 & 6.037427 & 8.3245 & 7.180964 \\
\hline KPNA2 & 11.226649 & 11.6355 & 11.43109 & 7.338309 & 7.8375 & 7.587905 \\
\hline COX7B & 12.179636 & 11.3544 & 11.76702 & 8.827969 & 8.235 & 8.531484 \\
\hline LIPA & 12.815084 & 11.6589 & 12.23699 & 9.059839 & 9.3255 & 9.19267 \\
\hline ZFP3 & 10.863089 & 12.4356 & 11.64934 & 10.46896 & 9.3255 & 9.897231 \\
\hline EIF4A2 & 12.342399 & 13.3479 & 12.98746 & 7.819575 & 5.2345 & 6.3456 \\
\hline TNFRSF12A & 10.364899 & 12.3245 & 11.3447 & 8.957959 & 8.3456 & 8.34566 \\
\hline MAP3K3 & 9.738604 & 9.23456 & 8.273484 & 8.09329 & 7.23455 & 6.238847 \\
\hline MAOB & 10.040855 & 13.7844 & 12.76466 & 9.658285 & 8.93774 & 7.377461 \\
\hline CCR5 & 10.378646 & 11.3765 & 10.34764 & 7.098104 & 8.38746 & 9.378645 \\
\hline E2F5 & 12.297534 & 13.3746 & 11.32374 & 10.19454 & 10.34662 & 9.37462 \\
\hline TOMM70A & 8.951378 & 8.3875 & 7.934875 & 11.35481 & 10.37468 & 9.376462 \\
\hline PDE6D & 9.847308 & 8.23675 & 7.374662 & 12.33905 & 10.38642 & 11.36525 \\
\hline DHX37 & 7.104071 & 7.23642 & 8.323786 & 8.647532 & 9.238765 & 9.236455 \\
\hline WDR93 & 7.613555 & 7.23765 & 8.232386 & 9.800161 & 10.37452 & 11.93478 \\
\hline HSBP1 & 9.246032 & 8.23885 & 8.23865 & 11.5736 & 10.37846 & 12.62356 \\
\hline DDX1 & 10.776629 & 10.2387 & 9.237658 & 12.12334 & 13.23775 & 11.76325 \\
\hline TSPO2 & 10.47408 & 8.32486 & 9.236746 & 11.80525 & 12.23877 & 11.23876 \\
\hline GPD2 & 12.100197 & 11.239 & 13.2384 & 14.55473 & 13.39845 & 14.23987 \\
\hline CYP27B1 & 13.075796 & 12.3896 & 11.93784 & 13.39113 & 14.23789 & 13.78467 \\
\hline STYXL1 & 10.783392 & 9.98746 & 10.35623 & 12.57094 & 11.23898 & 12.87364 \\
\hline GPR135 & 10.884162 & 10.3898 & 11.23876 & 11.55622 & 12.89348 & 13.38763 \\
\hline
\end{tabular}

HCC, hepatocellular carcinoma.

of development and progression of cancer, proliferation and differentiation serves an important role.

Numerous genes are significantly different between HCC and adjacent tissues. The top 22 differentially expressed genes were selected from 6 samples, then $\mathrm{MeV}$ version 4.8 (mev.tm4.org/) was used for hierarchical clustering (Fig. 2A). The cluster result demonstrated that the 3 HCC samples and 3 adjacent samples are divided into two different groups. This indicates that the expression of these genes can separate the HCC and adjacent samples by the hierarchical clustering method. In addition, the cluster result for the genes also generated 2 groups, the expression of genes of the first group in the HCC sample was greater than that of the adjacent samples. The features of these 12 genes may serve a specific role in HCC. The expression of additional genes was greater in the adjacent samples than in the HCC samples (Table I). In addition, the expression levels of cytochrome P450 family 27 subfamily B member 1 (CYP27B1) and glycerol-3-phosphate dehydrogenase 2 (GPD2) were high in the HCC and adjacent samples, suggesting that these two genes serve an important role in the two sample types. The results were in agreement with previous studies, for example, hydroxysteroid (11- $\beta$ ) dehydrogenase 1 (HSD11B1), the top upregulated genes in the HCC samples in the current study, was previously reported to be upregulated in patients with liver cancer $(16,17)$.

RT-qPCR was used to confirm the differences in gene expression (Fig. 2B). Among the 22 genes analyzed, 9 genes which have not been previously investigated were selected and were differently expressed in the microarray confirming the RT-qPCR findings. Among them, cyclooxygenase 7B (COX7B) has been reported to be overexpressed in nasopharyngeal carcinoma in a similar manner (18), as a previously known chemoresistance gene, COX7B also has been identified to be upregulated in post-chemotherapeutic ovarian tumors (19). WD repeat domain 93 (WDR93) which was identified to be downregulated in HCC tumor samples and was not previously associated with disease, was identified to be associated with neurological disorders through whole-exome sequencing (20). In controlling neutral lipid metabolism, liver homeostasis, immune response and tumor metastasis, lipase [also termed LAL, encoded by lipase A, lysosomal acid type (LIPA)] is a critical metabolic enzyme, which was identified in the current study to be upregulated. Tumor-promoting myeloid-derived suppressive cells in the liver of $\mathrm{LAL}^{-/-}$mice were reported to be reduced by human LIPA expression (21). TNFRSF12A expression in HCC tissue was detected to be 5.3 and 6.9 times higher than in adjacent tissue through RT-qPCR and microarray, respectively.

To illustrate the difference of TNFRSF12A gene expression levels between cancer and normal tissue, The Cancer Genome Atlas (TCGA) was used. TCGA is a comprehensive effort to accelerate understanding of the molecular basis of cancer through the use of genome analysis technologies, including large-scale genomic sequencing. Using TCGA, 3-level expression data was downloaded; the data contained 
A

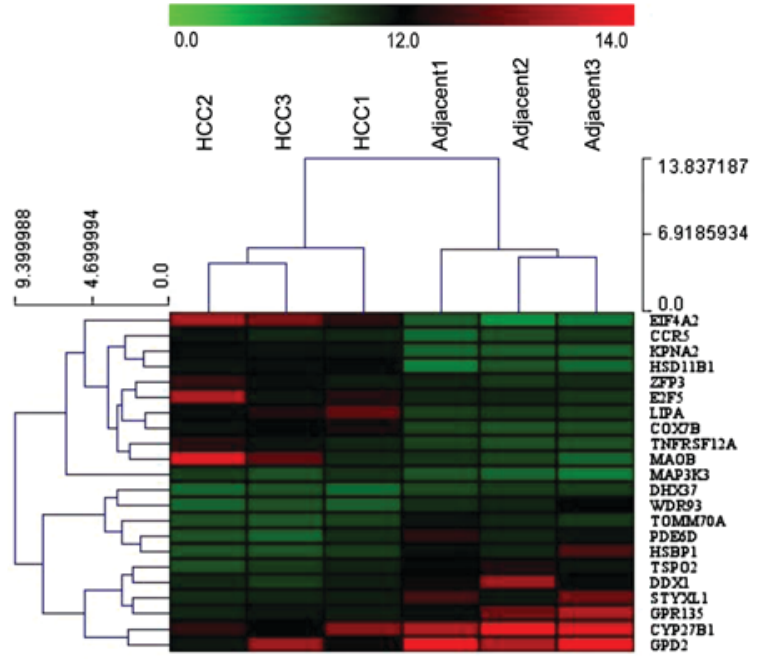

B

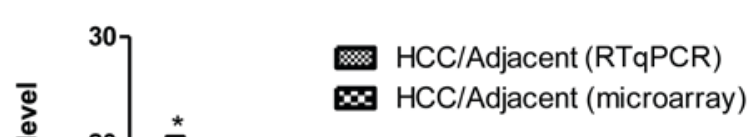

C Expression of TNFRSF12A in TCGA

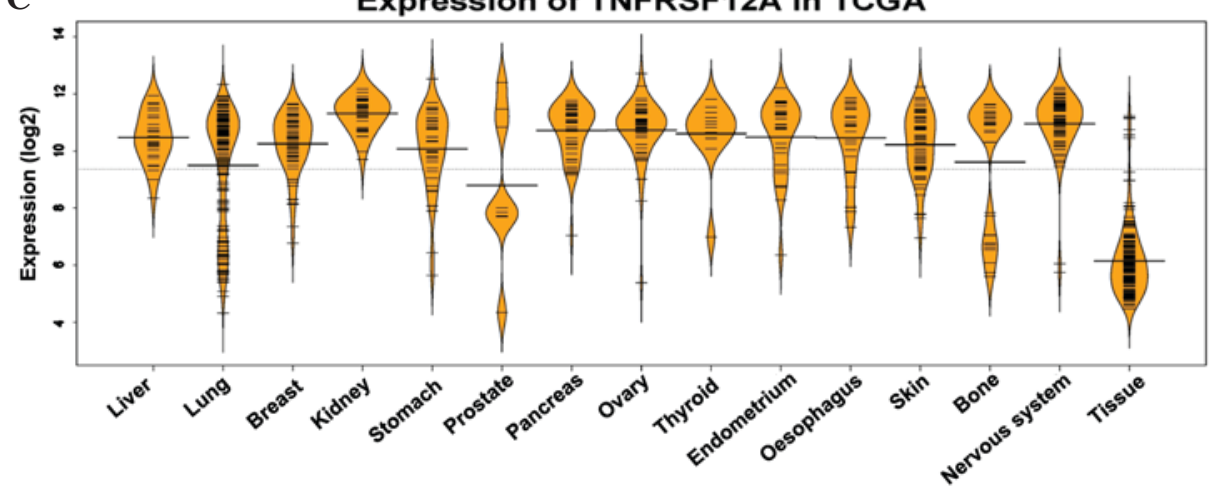

Figure 2. Gene expression levels between HCC and adjacent tissue. (A) Hierarchical clustering and heat map analysis of the top 22 genes. (B) Gene expression in HCC and adjacent samples detected by RT-qPCR and microarray. The $\Delta \Delta \mathrm{Cq}$ method was used to calculate the fold change in the expression of each gene and data were statistically analyzed using a two-tailed t-test. (C) Gene expression of TNFRSF12A in TCGA. ${ }^{*}<<0.05 ;{ }^{* *} \mathrm{P}<0.01$; ${ }^{* * *} \mathrm{P}<0.001 \mathrm{HCC}$ vs. adjacent tissue. HCC, hepatocellular carcinoma; RT-qPCR, reverse transcription-quantitative polymerase chain reaction; TNFRSF12A, tumor necrosis factor receptor superfamily member 12A; TCGA, The Cancer Genome Atlas.

A

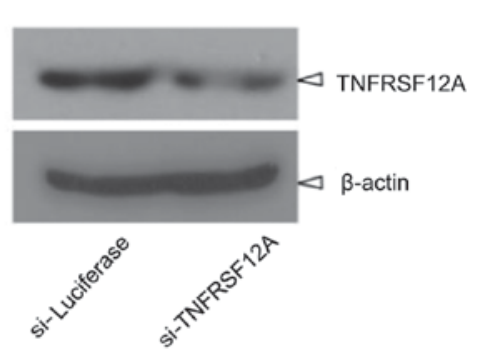

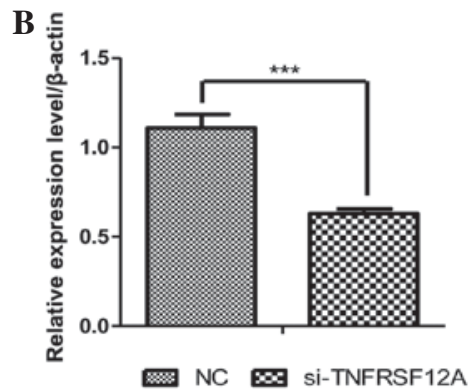

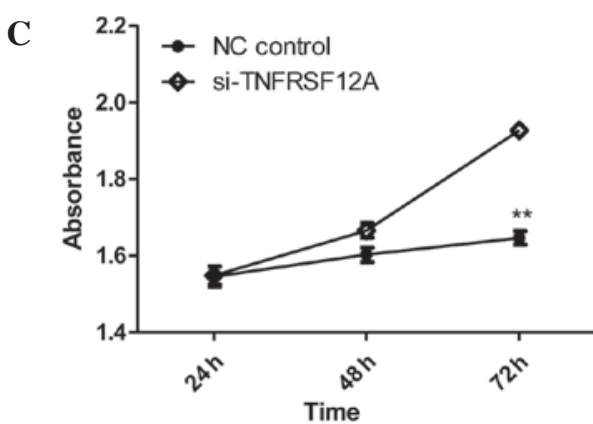

Figure 3. TNFRSF12A knockdown can reduce SMMC7721 cell viability. (A) TNFRSF12A expression detected in SMMC7721 cells following different siRNA transfection through western blotting, $\beta$-actin was used as the loading control. (B) Densitometric analysis was performed using ImageJ software to analyze TNFRSF12A expression. (C) Cell growth curves were increased according to cell viability for three days compared with NC siRNA transfection. ${ }^{* *} \mathrm{P}<0.01 ;{ }^{* * *} \mathrm{P}<0.001$ si-TNFRSF12A vs. NC control. TNFRSF12A, tumor necrosis factor receptor superfamily member 12A; NC, negative control; siRNA, small interfering RNA. 
A

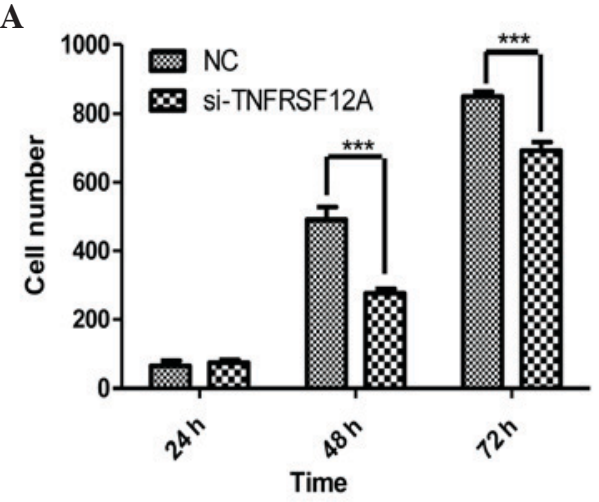

B

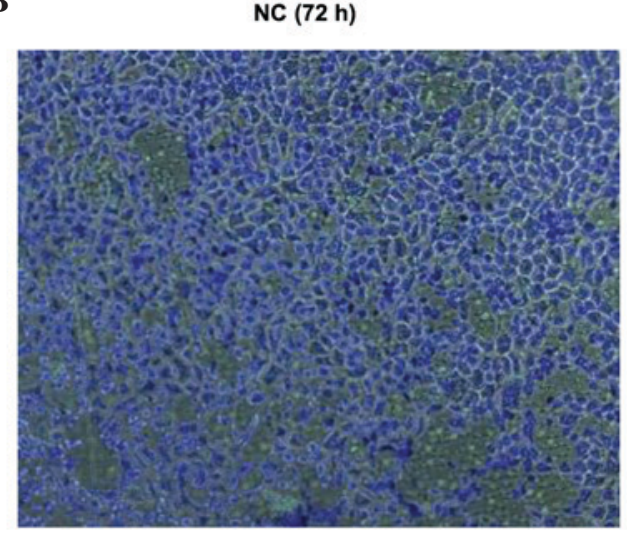

C

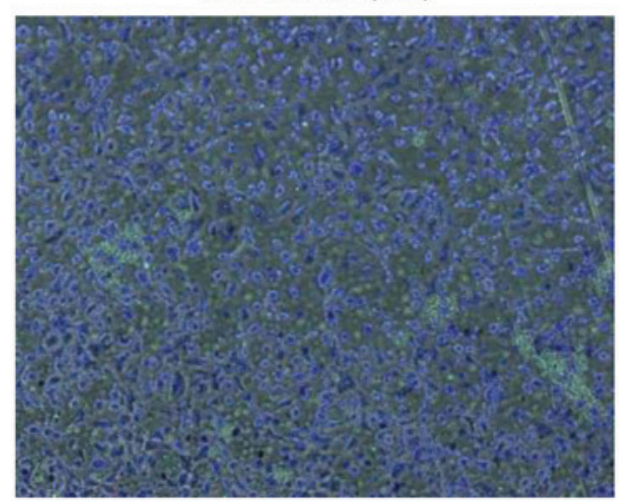

Figure 4. Effect of TNFRSF12A knockdown on migration and invasion of SMMC7721 cells. (A) Quantitative differences of cells between NC and si-TNFRSF12A transfection following transfection of different siRNAs and incubation for 24, 48 and $72 \mathrm{~h}$. (B and C) Representative images of the Transwell migration assay show the number of cells passing through the membrane was significantly reduced in the si-TNFSRF12A group compared with the NC group. Magnification, $\mathrm{x} 10 .{ }^{* * * *} \mathrm{P}<0.001$. TNFRSF12A, tumor necrosis factor receptor superfamily member 12A; NC, negative control; siRNA, small interfering RNA.

14 cancer sample types and 1 normal tissue sample type. The expression of TNFRSF12A in the cancer sample type was in general greater than that of the normal sample type (Fig. 2C), which was consistent with previous studies.

TNFRSF12A knockdown reduced SMMC7721 cell viability. TNFRSF12A, the smallest member of the TNF superfamily of receptors that lacks the cytoplasmic death domain (7), has been reported to be elevated in various types of cancer, including HCC, which was confirmed by clear upregulation in HCC tissues via microarray and RT-qPCR (Fig. 2 and Table I). In order to investigate the underlying molecular mechanisms in HCC, TNFRSF12A was selected for knockdown in the SMMC7721 cell line through siRNA. A total of $48 \mathrm{~h}$ after initial transfection, TNFRSF12A was detected to be downregulated through western blotting (Fig. 3A and B). Cell growth was measured 24, 48 and $72 \mathrm{~h}$ after initial siRNA transfection and according to the cell growth curves (Fig. 3C), cell viability was stronger in the si-TNFRSF12A transfected group in comparison with that of the NC-siRNA transfected group, suggesting that TNFRSF12A increases SMMC7721 cell viability in vitro.

TNFRSF12A knockdown can reduce invasive potential and metastatic capacity of SMMC7721 cells in vitro. Acting via cell-secreted proteolytic degradation of the cellular basement membrane, tumor cells invasion is the leading cause of cancer-associated mortality (22). Compared with the NC group, SMMC7721 cells which were transfected with si-TNFSFR12A exhibited greatly inhibited invasive potential and metastatic capacity. The number of cells passing through the membrane was significantly reduced in the si-TNFSRF12A group at 48 and $72 \mathrm{~h}$ (Fig. 4).

\section{Discussion}

HCC is a tumor type that is highly insensitive to conventional chemotherapy (23), and increasingly targeted molecular therapies have exhibited significant benefits in patients with cancer, including those with HCC. The eludication of the molecular mechanism of HCC occurrence and development is important for the development of effective treatments for HCC. Thus, in the current study, the Agilent Whole Human Genome Oligo Microarray ( $4 \times 44 \mathrm{~K}$ ) was used to investigate the differentially expressed genes between HCC and adjacent tissues, and the top 22 differentially expressed genes were confirmed by RT-qPCR. Among these large differences in gene expression, TNFRSF12A expression in HCC tissue was identified to be greater than that of adjacent tissue. The TNFRSF12A receptor has been previously identified to promote the invasive 
potential and metastatic capacity of non-small lung adenocarcinoma cells through the upregulation of integrin $\alpha 6$ (24). As the sole signaling receptor for the proinflammatory cytokine TWEAK (TNFSF12), TNFRSF12A engagement stimulates multiple signal transduction pathways, including the nuclear factor $\kappa \mathrm{B}$ pathway, and it has been previously identified that TNFRSF12A may serve a role in tumor growth and metastasis (25). in the current study, TNFRSF12A was knocked down in a SMMC7721 cell line through siRNA, and cells exhibited reduced reproductive and metastatic capacity ex vivo. Thus, the present study suggests that TNFRSF12A may be a candidate therapeutic target for cancer including $\mathrm{HCC}$, and significant differentially expressed genes between $\mathrm{HCC}$ and normal adjacent tissues require further investigation in subsequent studies.

\section{References}

1. Deng GL, Zeng S and Shen H: Chemotherapy and target therapy for hepatocellular carcinoma: New advances and challenges. World J Hepatol 7: 787-798, 2015.

2. Toyoda H, Kumada T, Tada T, Sone Y, Kaneoka Y and Maeda A Tumor markers for hepatocellular carcinoma: Simple and significant predictors of outcome in patients with HCC. Liver Cancer 4: 126-136, 2015.

3. Yu M, Tang Z, Meng F, Tai M, Zhang J, Wang R, Liu C and $\mathrm{Wu} \mathrm{Q}$ : Elevated expression of FoxM1 promotes the tumor cell proliferation in hepatocellular carcinoma. Tumour Biol 37: 1289-1297, 2016.

4. Wang Y and Tian Y: miRNA for diagnosis and clinical implications of human hepatocellular carcinoma. Hepatol Res 46: 89-99, 2016.

5. Tsang FH, Au SL, Wei L, Fan DN, Lee JM, Wong CC, Ng IO and Wong CM: MicroRNA-142-3p and microRNA-142-5p are downregulated in hepatocellular carcinoma and exhibit synergistic effects on cell motility. Front Med 9: 331-343, 2015.

6. Nishida $\mathrm{N}$ and Kudo M: Alteration of epigenetic profile in human hepatocellular carcinoma and its clinical implications. Liver Cancer 3: 417-427, 2014.

7. Wiley SR, Cassiano L, Lofton T, Davis-Smith T, Winkles JA, Lindner V, Liu H, Daniel TO, Smith CA and Fanslow WC: A novel TNF receptor family member binds TWEAK and is implicated in angiogenesis. Immunity 15: 837-846, 2001.

8. Li N, Hu WJ, Shi J, Xue J, Guo WX, Zhang Y, Guan DX, Liu SP, Cheng YQ, Wu MC, et al: Roles of fibroblast growth factor-inducible 14 in hepatocellular carcinoma. Asian Pac J Cancer Prev 14: 3509-3514, 2013.

9. Affò S, Dominguez M, Lozano JJ, Sancho-Bru P, Rodrigo-Torres D, Morales-Ibanez O, Moreno M, Millán C, Loaeza-del-Castillo A, Altamirano J, et al: Transcriptome analysis identifies TNF superfamily receptors as potential therapeutic targets in alcoholic hepatitis. Gut 62: 452-460, 2013.

10. Yin J, Liu YN, Tillman H, Barrett B, Hewitt S, Ylaya K, Fang L, Lake R, Corey E, Morrissey C, et al: AR-regulated TWEAK-FN14 pathway promotes prostate cancer bone metastasis. Cancer Res 74: 4306-4317, 2014.
11. Huang M, Narita S, Tsuchiya N, Ma Z, Numakura K, Obara T, Tsuruta H, Saito M, Inoue T, Horikawa Y, et al: Overexpression of Fn14 promotes androgen-independent prostate cancer progression through MMP-9 and correlates with poor treatment outcome. Carcinogenesis 32: 1589-1596, 2011.

12. Kwon OH, Kim JH, Kim SY and Kim YS: TWEAK/Fn14 signaling mediates gastric cancer cell resistance to 5-fluorouracil via NF-kB activation. Int J Oncol 44: 583-590, 2014.

13. Zhou H, Hittelman WN, Yagita H, Cheung LH, Martin SS, Winkles JA and Rosenblum MG: Antitumor activity of a humanized, bivalent immunotoxin targeting fn14-positive solid tumors. Cancer Res 73: 4439-4450, 2013.

14. Zhou H, Ekmekcioglu S, Marks JW, Mohamedali KA, Asrani K, Phillips KK, Brown SA, Cheng E, Weiss MB, Hittelman WN, et al: The TWEAK receptor Fn14 is a therapeutic target in melanoma: Immunotoxins targeting Fn14 receptor for malignant melanoma treatment. J Invest Dermatol 133: 1052-1062, 2013.

15. Livak KJ and Schmittgen TD: Analysis of relative gene expression data using real-time quantitative PCR and the 2(-Delta Delta C(T)) method. Methods 25: 402-408, 2001.

16. Pan ZQ, Fang ZQ and Lu WL: Characteristics of gene expression of adrenal cortical steroid synthetase and its regulatory factor in mice with $\mathrm{H} 22$ liver cancer of different patterns. Zhongguo Zhong Xi Yi Jie He Za Zhi 31: 85-89, 2011 (In Chinese).

17. Soldan M, Nagel G, Losekam M, Ernst M and Maser E: Interindividual variability in the expression and NNK carbonyl reductase activity of 11beta-hydroxysteroid dehydrogenase 1 in human lung. Cancer Lett 145: 49-56, 1999.

18. Xiong S, Wang Q, Zheng L, Gao F and Li J: Identification of candidate molecular markers of nasopharyngeal carcinoma by tissue microarray and in situ hybridization. Med Oncol 28 (Suppl 1): S341-S348, 2011.

19. L'Espérance S, Popa I, Bachvarova M, Plante M, Patten N, Wu L, Têtu B and Bachvarov D: Gene expression profiling of paired ovarian tumors obtained prior to and following adjuvant chemotherapy: Molecular signatures of chemoresistant tumors. Int J Oncol 29: 5-24, 2006.

20. Alazami AM, Patel N, Shamseldin HE, Anazi S, Al-Dosari MS, Alzahrani F, Hijazi H, Alshammari M, Aldahmesh MA, Salih MA, et al: Accelerating novel candidate gene discovery in neurogenetic disorders via whole-exome sequencing of prescreened multiplex consanguineous families. Cell Rep 10: 148-161, 2015.

21. Du H,Zhao T, Ding X and Yan C: Hepatocyte-Specific expression of human lysosome acid lipase corrects liver inflammation and tumor metastasis in lal(-/-) mice. Am J Pathol 185: 2379-2389, 2015.

22. Peng HX, Wu WQ, Yang DM, Jing R, Li J, Zhou FL, Jin YF, Wang SY and Chu YM: Role of B7-H4 siRNA in proliferation, migration, and invasion of LOVO colorectal carcinoma cell line. Biomed Res Int 2015: 326981, 2015.

23. Liang XD, Dai YC, Li ZY, Gan MF, Zhang SR, Yin-Pan, Lu HS, Cao XQ, Zheng BJ, Bao LF, et al: Expression and function analysis of mitotic checkpoint genes identifies TTK as a potential therapeutic target for human hepatocellular carcinoma. PLoS One 9: e97739, 2014.

24. Jandova J, Mason CJ, Pawar SC and Watts GS: Fn14 receptor promotes invasive potential and metastatic capacity of non-small lung adenocarcinoma cells through the up-regulation of integrin a6. Neoplasma 62: 41-52, 2015.

25. Cheng E, Whitsett TG, Tran NL and Winkles JA: The TWEAK receptor Fn14 Is an Src-inducible protein and a positive regulator of Src-driven cell invasion. Mol Cancer Res 13: 575-583, 2015. 